\title{
Thermal Control Using Surface Plasma Actuation
}

\author{
Subrata Roy \\ University of Florida, Gainesville, FL 32611, USA
}

Cooling of intricate surfaces are of great scientific and industrial importance. Common convective or conductive approaches have limited reach and due to their power and weight penalty are not very effective. Especially convective cooling methods include moving parts and thus suffer from reliability issues. As an alternative, plasma based cooling technologies are proposed as it can be very effective and are known for fast response even for hard to reach areas. They do not have any mechanical components, can be applied to the receptive locations and are generally surface complaint. Plasma devices can also be miniaturized to coat a surface. Examples of plasma based cooling include various non-linear electrode shapes like serpentine, horseshoe and needle. Specifically, we will show how these electrode geometries can improve the cooling effectiveness for turbine blades and electronic surfaces by over 50\% using very low power. These actuators also induce local three-dimensional vortices which may be used in boundary layer flows for separation control and drag reduction. 